Proyecciones Journal of Mathematics

Vol. 35, Nº 3, pp. 339-367, September 2016.

Universidad Católica del Norte

Antofagasta - Chile

\title{
Gliding Hump Properties in Abstract Duality Pairs with Projections
}

\author{
Charles Swartz \\ New Mexico State University, U.S.A. \\ Received : April 2016. Accepted : August 2016
}

\begin{abstract}
Let $E, G$ be Hausdorff topological vector spaces and let $F$ be a vector space. Assume there is a bilinear operator $\langle\cdot, \cdot\rangle: E \times F \rightarrow G$ such that $\langle\cdot, y\rangle: E \rightarrow G$ is continuous for every $y \in F$. The triple $E, F, G$ is called an abstract duality pair with respect to $G$ or an abstract triple and is denoted by $(E, F: G)$. If $\left\{P_{j}\right\}$ is a sequence of continuous projections on $E$, then $(E, F: G)$ is called an abstract triple with projections. Under appropriate gliding hump assumptions, a uniform bounded principle is established for bounded subsets of $E$ and pointwise bounded subsets of $F$. Under additional gliding hump assumptions, uniform convergent results are established for series $\sum_{j=1}^{\infty}\left\langle P_{j} x, y\right\rangle$ when $x$ varies over certain subsets of $E$ and $y$ varies over certain subsets of $F$. These results are used to establish uniform countable additivity results for bounded sets of indefinite vector valued integrals and bounded subsets of vector valued measures.
\end{abstract}




\section{Introduction}

In the paper [12], D. Noll and W. Stadler introduced an abstract notion of sectional operators modeled on the natural sectional projections of sequence spaces. They established a number of results for such sectional operators and gave applications to scalar sequence and function spaces. In [20] Zheng, Cui and Li gave a generalization of sectional operators to abstract duality pairs and established several uniform convergence results along with applications.

In this paper we present an abstraction of the coordinate projections for scalar and vector valued sequence spaces much in the spirit of the abstraction of sectional operators given by Zheng, Cui and $\mathrm{Li}([20])$. We establish a uniform boundedness principle for abstract duality pairs which generalizes a scalar uniform boundedness result of $\mathrm{Wu}$, Luo and Cui ([19]). We also establish several uniform convergence results analogous to those of Zheng, Cui and $\mathrm{Li}([20])$. In some sense our presentation requires fewer assumptions and offers simpler notation than that of sectional operators and the conclusions are stated in terms of series. Of course, it is possible to transfer back and forth between projection and sectional operators. We indicate several applications which give general uniform convergence results for vector valued sequence spaces, spaces of vector valued integrable functions and vector valued measures.

We first describe what is meant by an abstract duality pair. Let $E, G$ be Hausdorff topological vector spaces and let $F$ be a vector space. Assume there is a bilinear operator $\langle\cdot, \cdot\rangle: E \times F \rightarrow G$ such that $\langle\cdot, y\rangle: E \rightarrow G$ is continuous for every $y \in F$. The triple $E, F, G$ is called an abstract duality pair with respect to $G$ or an abstract triple and is denoted by $(E, F: G)$ (see [3], [20] for more general versions).

We assume throughout that $(E, F: G)$ is an abstract duality pair with respect to $G$ and that there exist a sequence of projection operators $\left\{P_{j}\right\}$ , $P_{j}: E \rightarrow E$, which are continuous with respect to the topology of $E$. We refer to the triple $E, F, G$ and the projections $\left\{P_{j}\right\}$ as an abstract duality pair or an abstract triple with projections. One can construct sectional

operators $s_{k}$ as in [12],[19],[20] by setting $s_{k}=\sum_{j=1}^{k} P_{j}$; if one assumes $P_{i} P_{j}=0$ when $i \neq j$, then the sectional operators will satisfy $s_{k} s_{l}=s_{l}$ if $l \leq$ $k$ as required in [12],[19],[20]. It should be noted that these authors require that the space $F$ is also equipped with sectional operators, an assumption we do not make.

We give examples below. 
Example 1. Of course, the simplest example of an abstract duality pair is just a pair of vector spaces $E, F$ in duality where $G$ is the space of scalars. Here, $E$ can be a locally convex space and $F$ its dual or $E$ can carry the weak topology $\sigma(E, F)$ on $E$ induced by $F$.

We give examples of abstract duality pairs with projections.

Example 2. Let $\lambda$ be a scalar valued sequence space which contains $c_{00}$, the space of all scalar sequences which are eventually 0 . If $t \in \lambda$, we write $t=\left\{t_{j}\right\}$ so $t_{j}$ is the $j^{\text {th }}$ coordinate of $t$. The $\beta$-dual, $\lambda^{\beta}$, of $\lambda$ is

$$
\lambda^{\beta}=\left\{\left\{s_{j}\right\}: \sum_{j=1}^{\infty} s_{j} t_{j} \text { converges for every } t \in \lambda\right\} .
$$

Then $\lambda, \lambda^{\beta}$ form a dual pair with respect to the pairing $t \cdot s=\sum_{j=1}^{\infty} s_{j} t_{j}$. Let $e^{j}$ be the sequence with 1 in the $j^{\text {th }}$ coordinate and 0 in the other coordinates. Then for every $j \in \mathbf{N}, P_{j}(t)=t_{j} e^{j}$ defines the coordinate projection $P_{j}: \lambda \rightarrow \lambda$ from $t$ onto its $j^{\text {th }}$ coordinate. Each $P_{j}$ is obviously $\sigma\left(\lambda, \lambda^{\beta}\right)$ continuous and in many cases $\lambda$ will carry a locally convex topology with respect to which each $P_{j}$ will be continuous.

More generally, we have

Example 3. Let $X, Y$ be topological vector spaces and let $E$ be a vector space of $X$ valued sequences which contains $c_{00}(X)$, the space of all $X$ valued sequences which are eventually 0 . Let $L(X, Y)$ be the space of all continuous linear operators from $X$ into $Y$. The $\beta$-dual of $E$ with respect to $Y, E^{\beta Y}$, is defined to be

$E^{\beta Y}=\left\{\left\{T_{j}\right\} \subset L(X, Y): \sum_{j=1}^{\infty} T_{j} x_{j}=x \cdot T\right.$ converges for every $\left.x=\left\{x_{j}\right\} \in E\right\}$

and we have a bilinear operator $(x, T) \rightarrow x \cdot T$ from $E \times E^{\beta Y} \rightarrow Y$. If $w\left(E, E^{\beta Y}\right)$ is the weakest topology on $E$ such that all the linear maps $x \rightarrow x \cdot T$ from $E$ into $Y$ are continuous for every $T \in E^{\beta Y}$, then $E, E^{\beta Y}$ is an abstract duality pair with respect to $Y,\left(E, E^{\beta Y}: Y\right)$. If $z \in X$ and $j \in \mathbf{N}$, let $e^{j} \otimes z$ be the sequence with $z$ in the $j^{\text {th }}$ coordinate and 0 in the other coordinates. The space $E$ has the natural coordinate projection operators $P_{j}$ defined by $P_{j} x=e^{j} \otimes x_{j}$ which are continuous with respect to $w\left(E, E^{\beta Y}\right)$. Then $E, E^{\beta Y}$ is an abstract duality pair with respect to $Y$ with projections. 
We give several non-sequence space examples. Let $(S, \Sigma, \mu)$ be a $\sigma$-finite measure space with $\left\{A_{j}\right\}$ a pairwise disjoint sequence from $\Sigma$.

Example 4. Then $L^{1}(\mu), L^{\infty}(\mu)$ form a dual pair under the bilinear map $(f, g)=\int_{S} f g d \mu$ which is continuous with respect to the natural topologies and $P_{j} f=\chi_{A_{j}} f$ defines a projection operator on $L^{1}(\mu)$ which is $\|\cdot\|_{1}$ continuous, where $\chi_{A}$ is the characteristic function of $A$.

More generally, let $X$ be a Banach space.

Example 5. Let $L^{1}(\mu, X)$ be the space of all $X$ valued, Bochner $\mu$ integrable functions with the $L^{1}$ norm $\|f\|_{1}=\int_{S}\|f(s)\| d \mu(s)$ ([2]5.2, [4]II.2). Then $\langle f, g\rangle=\int_{S} g(s) f(s) d \mu(s)$ defines a continuous bilinear operator $\langle\cdot, \cdot \cdot$ : $L^{1}(\mu, X) \times L^{\infty}(\mu) \rightarrow X$ when $L^{\infty}(\mu)$ has its natural topology and $P_{j} f=$ $\chi_{A_{j}} f$ defines a continuous projection operator on $L^{1}(\mu, X)$. Then $\left(L^{1}(\mu, X), L^{\infty}(\mu)\right.$ : $X)$ is an abstract duality pair with respect to $X$ with projections. Similarly, if $L^{\infty}\left(\mu, X^{\prime}\right)$ is the space of essentially $\mu$-bounded, $X^{\prime}$ valued functions with its natural topology, $\left(L^{1}(\mu, X), L^{\infty}\left(\mu, X^{\prime}\right)\right)$ is a dual pair with projections $\left\{P_{j}\right\}$. Dually,

$$
\left(L^{\infty}(\mu), L^{1}(\mu, X): X\right),\left(L^{\infty}(\mu, X), L^{1}\left(\mu, X^{\prime}\right)\right) \text { and }\left(L^{\infty}(\mu), L^{1}(\mu)\right)
$$

are abstract triples with the projections defined as above.

Similarly,

Example 6. Let $D(\mu, X)[P(\mu, X)]$ be the space of $X$ valued Dunford [Pettis] $\mu$ integrable functions with the norm

$$
\|f\|_{D}=\sup \left\{\int_{S}\left|x^{\prime} f\right| d \mu:\left\|x^{\prime}\right\| \leq 1\right\}
$$

(see [2]5.2,[4]II.3, for these integrals). Then $\langle f, g\rangle=\int_{S} g(s) f(s) d \mu(s)$ defines a continuous bilinear operator $\langle\cdot, \cdot\rangle: D(\mu, X) \times L^{\infty}(\mu) \rightarrow X^{\prime \prime}[\langle\cdot, \cdot\rangle$ : $\left.P(\mu, X) \times L^{\infty}(\mu) \rightarrow X\right]\left(\right.$ if $x^{\prime} \in X^{\prime},\left\|x^{\prime}\right\| \leq 1$,

$$
\left|x^{\prime} \int_{S} g(s) f(s) d \mu(s)\right|=\left|\int_{S} g(s) x^{\prime} f(s) d \mu(s)\right| \leq\|g\|_{\infty} \int_{S}\left|x^{\prime} f(s)\right| d \mu(s)
$$

so $\|\langle f, g\rangle\| \leq\|f\|_{D}\|g\|_{\infty}$; for the integrability of $g f$ in the Pettis intregrable case, see [4]II.3.8). Also $P_{j} f=\chi_{A_{j}} f$ defines a continuous projection operator on $D(\mu, X)[P(\mu, X)]$. Then 


$$
\left(D(\mu, X), L^{\infty}(\mu): X^{\prime \prime}\right)\left[\left(P(\mu, X), L^{\infty}(\mu): X\right)\right]
$$

is an abstract duality with respect to $X^{\prime \prime}[X]$ with projections. Dually,

$$
\left(L^{\infty}(\mu), D(\mu, X): X^{\prime \prime}\right)\left[\left(L^{\infty}(\mu), P(\mu, X): X\right)\right]
$$

is an abstract triple with the projections defined similarly.

Note in all of the examples above the projections satisfy $P_{i} P_{j}=0$ when $i \neq j$ so we can define sectional operators $s_{k}=\sum_{j=1}^{k} P_{j}$ which satisfy $s_{k} s_{l}=s_{k}$ when $k \leq l$ as in [12],[19],[20].

We will give further examples of abstract triples with spaces of measures later.

We now define the $\beta$-dual with respect to an abstract duality pair with projections.

Definition 7. The $\beta$-dual of $E$ with respect to the abstract duality pair $(E, F: G)$ and projections $\left\{P_{j}\right\}$ is defined to be

$$
E^{\beta}=\left\{y \in F: \sum_{j=1}^{\infty}\left\langle P_{j} x, y\right\rangle \text { converges in } G \text { for every } x \in E\right\} \text {. }
$$

We write $x \cdot y=\sum_{j=1}^{\infty}\left\langle P_{j} x, y\right\rangle$ when $x \in E$ and $y \in E^{\beta}$ and we set $P_{I}=\sum_{j \in I} P_{j}$ when $I$ is a finite subset of $\mathbf{N}$.

A few remarks are in order. First, the $\beta$-dual of $E$ is a subset of $F$ and our notation does not reflect the dependence of the $\beta$-dual on the abstract duality pair with projections; hopefully, the statements will be clear from the context. It is also the case that the $\beta$-dual can be a proper subset of $F$. To see this consider the abstract triple $\left(l^{\infty}, D\left(\mu, c_{0}\right): l^{\infty}\right)$ of Example 6 , where $S=\mathbf{N}$ and $\mu$ is counting measure. Define $f: \mathbf{N} \rightarrow c_{0}$ by $f(j)=e^{j}$. Then $f$ is Dunford integrable (but not Pettis integrable) so $f \in D\left(\mu, c_{0}\right)$ and if $t=\left\{t_{j}\right\}^{\infty}, \int_{A} t f d \mu=\chi_{A} t$ [coordinate product]. Let $P_{j}$ be the coordinate projections as defined in Example 2 so $\left(l^{\infty}, D\left(\mu, c_{0}\right): l^{\infty}\right)$ is an abstract triple with projections. Let $e=\{1\}$ be the constant sequence of 1 's. Then

$$
\sum_{j=1}^{\infty}\left\langle P_{j} e, f\right\rangle=\sum_{j=1}^{\infty} \int_{\mathbf{N}} P_{j} e f d \mu=\sum_{j=1}^{\infty} e^{j}
$$


a series which does not converge in $l^{\infty}$ with respect to $\|\cdot\|_{\infty}$. Hence, $f \notin$ $\left(l^{\infty}\right)^{\beta}$ with respect to this abstract triple.

We say that $E$ is a weak AK space if for each $x \in E, x=\sum_{j=1}^{\infty} P_{j} x$, where the series is convergent with respect to $w\left(E, E^{\beta}\right)$. If $E$ is a weak AK space, then we have $E^{\beta}=F$ so this is a sufficient condition for equality between $F$ and the $\beta$ dual.

Next, we should compare this definition with the previous definitions of $\beta$-duals.

If $\lambda$ is a scalar sequence space as in Example 2 , then the definition in Example 2 above coincides with the definition above when we consider the abstract duality pair $\lambda, \lambda^{\beta}$ with respect to the scalar field and the projections defined in Example 2. To see the dependence of the $\beta$-dual on the abstract duality pair consider the space $c_{c}$ of scalar sequences which are eventually constant. In the duality pair $c_{c}, l^{1}$ the $\beta$-dual in this pair is $l^{1}$ while the $\beta$-dual in the classical setting of sequence spaces is $c s$, the space of convergent series $([7])$.

If $X, Y, E$ are as in Example 3 , then the $\beta$-dual as defined in Example 3 coincides with the definition above when we consider the abstract duality pair $E, E^{\beta Y}$ with respect to $Y$ and the projections defined in Example 3.

In Examples 4,5 and Example 6 for the Pettis integral, the $\beta$-dual is just $L^{\infty}(\mu)$. This follows from the countable additivity of the integrals in each case. In each case we would have $\sum_{j=1}^{\infty}\left\langle P_{j} f, g\right\rangle=\sum_{j=1}^{\infty} \int_{A_{j}} g f d \mu=$ $\int_{\cup_{j=1}^{\infty} A_{j}} g f d \mu$ for $g \in L^{\infty}(\mu)$ ([4] II.3.5).

We now consider one of our main results which depends on a gliding hump assumption. An interval $I$ in $\mathbf{N}$ is a subset of the form $I=\{j \in$ $\mathbf{N}: m \leq j \leq n\}, m \leq n$; a sequence of intervals $\left\{I_{j}\right\}$ is increasing if $\max I_{j}<\min I_{j+1}$. Let $w\left(E, E^{\beta}\right)$ be the weakest topology on $E$ such that the linear maps $x \rightarrow x \cdot y=\sum_{j=1}^{\infty}\left\langle P_{j} x, y\right\rangle$ are continuous from $E$ into $G$ for every $y \in E^{\beta}$.

Definition 8. The space $E$ (or the triple $(E, F: G)$ ) has the zero gliding hump property (0-GHP) if whenever $x^{k} \rightarrow 0$ in $E$ and $\left\{I_{k}\right\}$ is an increasing sequence of intervals, there is a subsequence $\left\{n_{k}\right\}$ such that the series $\sum_{k=1}^{\infty} P_{I_{n_{k}}} x^{n_{k}}$ is $w\left(E, E^{\beta}\right)$ convergent in $E$.

In the case when $E$ is a sequence space, in the usual definition of the $\beta$ dual the series $\sum_{k=1}^{\infty} P_{I_{n_{k}}} x^{n_{k}}$ in the definition above is required to converge pointwise or coordinatewise. Of course, in this abstract setting there is no notion of pointwise convergence; in this case there are 2 natural choices for 
the convergence of the series $\sum_{k=1}^{\infty} P_{I_{n_{k}}} x^{n_{k}}$, namely the topology $w\left(E, E^{\beta}\right)$ or the original topology of $E$.. We have chosen the topology $w\left(E, E^{\beta}\right)$ because it is often the weaker topology and it seems to be the correct topology for the proof of Theorem 13. Also, if $\lambda$ is a scalar sequence space and if the series $\sum_{k=1}^{\infty} P_{I_{n_{k}}} x^{n_{k}}$ is coordinatewise convergent to an element $x \in \lambda$, then the series is $\sigma\left(\lambda, \lambda^{\beta}\right)$ convergent to $x$ so we have agreement with the definition above. (The $\beta$-dual of a $\mathrm{K}$-space is often contained in the topological dual so the weak topology $w\left(E, E^{\beta}\right)$ is weaker than the original topology (see [7], page 68, for examples).) In the papers [WCL] and [20], the authors have chosen the original topology of $E$ and used sectional operators.

Examples of sequence spaces with 0-GHP can be found in [16], Appendices $\mathrm{B}$ and $\mathrm{C}$; further examples of function and measure spaces with 0-GHP will be given later. The concept of 0-GHP was introduced by Lee Peng Yee ([9]).

If $E$ is a Banach space having projections $\left\{P_{j}\right\}$ which satisfy the condition that $P_{i} P_{j}=0$ when $i \neq j$ and $\sup \left\{\left\|P_{I}\right\|: I \subset \mathbf{N}\right.$ finite $\}=M<\infty$ and if $Y$ is any locally convex space and $F \subset L(E, Y)$, then $(E, F: Y)$ is an abstract triple under the map $(x, T) \rightarrow T x$ and $E$ has 0-GHP. For if $x^{k} \rightarrow 0$ in $E$ and $\left\{I_{k}\right\}$ is an increasing sequence of intervals, there is a subsequence $\left\{n_{k}\right\}$ such that $\sum_{k=1}^{\infty}\left\|x^{n_{k}}\right\|<\infty$ and then

$$
\sum_{k=1}^{\infty}\left\|P_{I_{n_{k}}} x^{n_{k}}\right\| \leq M \sum_{k=1}^{\infty}\left\|x^{n_{k}}\right\|<\infty
$$

and the series $\sum_{k=1}^{\infty} P_{I_{n_{k}}} x^{n_{k}}$ converges to some $x \in E$ by completeness. But then the series is $w(E, F)$ convergent to $x$. More generally, any $K$ space where the projections satisfy the conditions above has 0-GHP (see [15] for the definition of $K$ space and examples; there exist non-complete normed $K$ spaces). In particular, these remarks give the following examples of triples with 0-GHP.

Example 9. $\left(L^{1}(\mu, X), L^{\infty}(\mu): X\right),\left(L^{\infty}(\mu), L^{1}(\mu, X): X\right)$ and the other triples in Example 5 have 0-GHP. As another example, let $1<p<\infty$ and $\frac{1}{p}+\frac{1}{q}=1$ and $L^{p}(\mu, X)$ be the space of strongly measurable functions which are $p^{\text {th }}$ power integrable with the norm $\|f\|_{p}=\left(\int_{S}\|f(s)\|^{p} d \mu(s)\right)^{1 / p}$. Then $\left(L^{p}(\mu, X), L^{q}\left(\mu, X^{\prime}\right)\right)$ form a dual pair under the pairing $\langle f, g\rangle=$ $\int_{S}\langle f(s), g(s)\rangle d \mu(s)$ and if $\left\{A_{j}\right\}$ is a pairwise disjoint sequence from $\Sigma$, then $P_{j} f=\chi_{A_{j}} f$ defines a sequence of continuous projections satisfying the condition above and $\left(L^{p}(\mu, X), L^{q}\left(\mu, X^{\prime}\right)\right)$ has $0-G H P$. 
Example 10. Similarly the triple $\left(L^{\infty}(\mu), P(\mu, X): X\right)$ of Example 6 has $0-G H P$.

As a dual result, we have

Example 11. The triple $\left(P(\mu, X), L^{\infty}(\mu): X\right)$ and the projections of Example 6 has 0-GHP. (Recall that $P(\mu, X)$ is not complete so the remark above does not apply ([2] Example 5.13, [5]).) Suppose $\left\{f_{k}\right\} \subset P(\mu, X)$ converges to 0 and for convenience assume $\left\|f_{k}\right\|<1 / 2^{k}$. Let $\left\{I_{k}\right\}$ be an increasing sequence of intervals and set $B_{k}=\cup_{j \in I_{k}} A_{j}$. Let $f$ be the pointwise limit of the series

$$
\sum_{k=1}^{\infty} P_{I_{k}} f_{k}=\sum_{k=1}^{\infty} \chi_{B_{k}} f_{k} .
$$

We claim that $f \in P(\mu, X)$. Obviously $f$ is scalarly measurable and if $x^{\prime} \in X^{\prime}$, then $x^{\prime} f=\sum_{k=1}^{\infty} x^{\prime} \chi_{B_{k}} f_{k}$ and $\left|x^{\prime} f\right|=\sum_{k=1}^{\infty}\left|x^{\prime} \chi_{B_{k}} f_{k}\right|$ pointwise. By the Monotone Convergence Theorem,

$$
\int_{S}\left|x^{\prime} f\right| d \mu=\sum_{k=1}^{\infty} \int_{B_{k}}\left|x^{\prime} f_{k}\right| d \mu \leq\left\|x^{\prime}\right\| \sum_{k=1}^{\infty}\left\|f_{k}\right\| \leq\left\|x^{\prime}\right\| \sum_{k=1}^{\infty} 1 / 2^{k}
$$

so $f$ is scalarly integrable. We claim that $f$ is Pettis integrable. Let $A \in \Sigma$. Since

$$
\sum_{k=1}^{\infty}\left\|\int_{A \cap B_{k}} f_{k} d \mu\right\| \leq \sum_{k=1}^{\infty}\left\|f_{k}\right\| \leq \sum_{k=1}^{\infty} 1 / 2^{k},
$$

the series $\sum_{k=1}^{\infty} \int_{A \cap B_{k}} f_{k} d \mu$ converges to some $x_{A} \in X$ since $X$ is complete. Now $x^{\prime}\left(x_{A}\right)=\sum_{k=1}^{\infty} \int_{A \cap B_{k}} x^{\prime} f_{k} d \mu$ and $\left|x^{\prime} f\right| \geq\left|\sum_{k=1}^{n} \chi_{A \cap B_{k}} f_{k}\right|$ for $n \in \mathbf{N}$ and $x^{\prime} \in X^{\prime}$ so the Dominated Convergence Theorem implies

$$
\int_{A} x^{\prime} f d \mu=\sum_{k=1}^{\infty} \int_{A \cap B_{k}} x^{\prime} f_{k} d \mu .
$$
Then

Hence $x_{A}=\int_{A} f d \mu \in X$ and $f$ is Pettis integrable. Let $g \in L^{\infty}(\mu)$.

$$
\begin{aligned}
\left\|g f-\sum_{k=1}^{n} g \chi_{B_{k}} f_{k}\right\| & =\sup \left\{\int_{S}\left|x^{\prime} \sum_{k=n+1}^{\infty} g f_{k}\right| d \mu:\left\|x^{\prime}\right\| \leq 1\right\} \\
& \leq \sup \left\{\sum_{k=n+1}^{\infty} \int_{B_{k}}\left|x^{\prime} g f_{k}\right| d \mu:\left\|x^{\prime}\right\| \leq 1\right\} \leq \sum_{k=n+1}^{\infty}\left\|f_{k}\right\|\|g\|_{\infty}
\end{aligned}
$$

so the series $\sum_{k=1}^{\infty} \chi_{B_{k}} f_{k}$ is $w\left(P(\mu, X), L^{\infty}(\mu)\right)$ convergent to $f$. Hence, the triple has $0-G H P$. 
Example 12. We give an example of a non-complete normed space whose projections satisfy the conditions above and which has 0-GHP. Consider Example 5 and let $Y$ be a subspace of $X$ and let $L^{1}(\mu, Y)$ be the subspace of $L^{1}(\mu, X)$ which consists of those functions with values in $Y$. Suppose $\left\{f_{k}\right\} \rightarrow 0$ in $L^{1}(\mu, Y)$ and $\left\{I_{k}\right\}$ is an increasing sequence of intervals. Note each $P_{I_{k}}$ is a projection of norm 1 and $P_{i} P_{j}=0$ for $i \neq j$. There is a subsequence $\left\{n_{k}\right\}$ such that $\sum_{k=1}^{\infty}\left\|f_{n_{k}}\right\|_{1}<\infty$. Then the series $\sum_{k=1}^{\infty} P_{I_{n_{k}}} f_{n_{k}}$ is absolutely convergent in $L^{1}(\mu, X)$ and the series $\sum_{k=1}^{\infty} P_{I_{n_{k}}} f_{n_{k}}$ is pointwise convergent to a function $f$ with values in $Y$ and the series is $w\left(L^{1}(\mu, Y), L^{\infty}(\mu)\right)$ convergent to $f$. Thus, the triple $\left(L^{1}(\mu, Y), L^{\infty}(\mu): X\right)$ has $0-G H P$.

We now establish the first of our main results which is a uniform boundedness result. For this and later results we use a signed version of a result of Antosik and Mikusinski for infinite matrices. An infinite matrix $M=\left[m_{p q}\right]$ with values in a topological vector space $Z$ is a signed $K$ matrix if the columns of $M$ converge in $Z$ and every increasing sequence of integers $\left\{m_{q}\right\}$ has a further subsequence $\left\{n_{q}\right\}$ and there exists a sequence of signs $\left\{s_{q}\right\}$ such that the series $\sum_{q=1}^{\infty} s_{q} m_{p n_{q}}$ converges and $\lim _{p} \sum_{q=1}^{\infty} s_{q} m_{p n_{q}}$ exists in $Z$. The signed version of the Antosik-Mikusinski Theorem asserts that the diagonal of $M,\left\{m_{p p}\right\}$, converges to 0 (see [15], [16] for this and more general versions of the theorem; the signed version of the theorem is due to Stuart ([14])). If all of the signs can be chosen equal to 1, the matrix $M$ is called a $K$ matrix.

Theorem 13. Assume that $E$ has $0-G H P$ and for every $j, P_{j} E$ is barrelled under the topology of $E$. If $A \subset E$ is bounded and $B \subset E^{\beta}$ is pointwise bounded on $E$, then $\left\{x \cdot y=\sum_{j=1}^{\infty}\left\langle P_{j} x, y\right\rangle: y \in B, x \in A\right\}$ is bounded in $G$.

Proof. If the conclusion fails to hold, there exist a closed symmetric neighborhood of $0, U$, in $G,\left\{y^{k}\right\} \subset B,\left\{x^{k}\right\} \subset A, 0<s_{k} \rightarrow 0$ such that $x^{k} \cdot s_{k} y^{k} \notin U$. Pick a closed, symmetric neighborhood of $0, V$, such that $V+V \subset U$. For $k_{1}=1$ pick $m_{1}$ such that $s_{k_{1}} \sum_{j=1}^{m_{1}}\left\langle P_{j} x^{k_{1}}, y^{k_{1}}\right\rangle \notin$ $U$. By the continuity of the $P_{j}, P_{j} A$ is bounded in $E$ with respect to the topology of $E,\left\langle\cdot, y^{k}\right\rangle: P_{j} E \rightarrow G$ is continuous with respect to the topologies of $E$ and $G$ and $\left\{\left\langle\cdot, y^{k}\right\rangle: k \in \mathbf{N}\right\}$ is pointwise bounded on $P_{j} E$. Since $P_{j} E$ is barrelled, for every $j,\left\{P_{j} x^{k} \cdot y^{k}: k\right\}$ is bounded in $G$. Therefore, $\lim _{k} s_{k}\left\langle P_{j} x^{k}, y^{k}\right\rangle=0$ in $G$ for every $j$. Hence, there exists 
$k_{2}>k_{1}$ such that $s_{k} \sum_{j=1}^{m_{1}}\left\langle P_{j} x^{k}, y^{k}\right\rangle \in V$ for $k \geq k_{2}$. Pick $m_{2}>m_{1}$ such that $s_{k_{2}} \sum_{j=1}^{m_{2}}\left\langle P_{j} x^{k_{2}}, y^{k_{2}}\right\rangle \notin U$. Put $I_{2}=\left[m_{1}+1, m_{2}\right]$. Then

$$
s_{k_{2}} \sum_{j \in I_{2}}\left\langle P_{j} x^{k_{2}}, y^{k_{2}}\right\rangle=s_{k_{2}} \sum_{j=1}^{m_{2}}\left\langle P_{j} x^{k_{2}}, y^{k_{2}}\right\rangle-s_{k_{2}} \sum_{j=1}^{m_{1}}\left\langle P_{j} x^{k_{2}}, y^{k_{2}}\right\rangle \notin V .
$$

Continuing this construction produces an increasing sequence $\left\{k_{p}\right\}$ and an increasing sequence of intervals $\left\{I_{p}\right\}$ such that

$$
\text { (\#) } s_{k_{p}} \sum_{j \in I_{p}}\left\langle P_{j} x^{k_{p}}, y^{k_{p}}\right\rangle \notin V \text {. }
$$

Define an infinite matrix

$$
M=\left[m_{p q}\right]=\left[\sqrt{s_{k_{p}}} \sum_{l \in I_{q}}\left\langle\sqrt{s_{k_{q}}} P_{l} x^{k_{q}}, y^{k_{p}}\right\rangle\right]=\left[\sqrt{s_{k_{p}}}\left\langle\sum_{l \in I_{q}} \sqrt{s_{k_{q}}} P_{l} x^{k_{q}}, y^{k_{p}}\right\rangle\right] .
$$

We claim that $M$ is a $K$ matrix. First, the columns of $M$ converge to 0 since $\left\{y^{p}\right\}$ is pointwise bounded on $E$. Next, since $\sqrt{s_{k_{q}}} x^{k_{q}} \rightarrow 0$ in $E$, the 0 -GHP implies that given any subsequence there is a further subsequence $\left\{r_{q}\right\}$ such that

$$
x=\sum_{q=1}^{\infty} \sqrt{s_{k_{r_{q}}}} \sum_{l \in I_{r_{q}}} P_{l} x^{k_{r_{q}}} \in E,
$$

where the series converges in $w\left(E, E^{\beta}\right)$. By the $w\left(E, E^{\beta}\right)$ convergence of the series for $x$, we have

$$
\sum_{q=1}^{\infty} m_{p r_{q}}=\sum_{q=1}^{\infty} \sqrt{s_{k_{p}}}\left\langle\sqrt{s_{k_{r_{q}}}} \sum_{l \in I_{r_{q}}} P_{l} x^{k_{r_{q}}}, y^{k_{p}}\right\rangle=x \cdot \sqrt{s_{k_{p}}} y^{k_{p}}
$$

and $x \cdot \sqrt{s_{k_{p}}} y^{k_{p}} \rightarrow 0$ since $\left\{y^{p}\right\}$ is pointwise bounded on $E$. Therefore, $M$ is a $K$ matrix and by the Antosik-Mikusinski Matrix Theorem the diagonal of $M$ converges to 0 . But, this contradicts (\#).

Remark 14. The proof of Theorem 13 shows that the assumption in Theorem 13 that the spaces $P_{j} E$ are barrelled can be replaced by the assumption that these are $A$ spaces; $A$ spaces have the property that pointwise bounded families of continuous linear operators on these spaces are uniformly bounded on bounded subsets (see [10], [15] for the definition and properties). 
The scalar version of Theorem 13 when $E, F$ is a dual pair of vector spaces gives a generalization of one part of Theorem 1 of [19] where it is assumed that $E$ is a normed AK space. See also Theorem 8.13 of [16] for a scalar sequence space version of the result.

We give an application of Theorem 13 to vector valued sequence spaces as in Example 3.

Example 15. Assume the notation as in Example 3 and further assume that $E$ has $0-G H P$ with respect to a locally convex topology and that the projections $P_{j} x=e^{j} \otimes x_{j}$ are continuous. If the space $X$ is barrelled (an $A$ space) and if the spaces $X$ and $P_{j} E=e^{j} \otimes X$ are isomorphic, Theorem 13 (Remark 14) implies that if $A \subset E$ is bounded in $E$ and $B \subset E^{\beta Y}$ is pointwise bounded on $E$, then $\left\{x \cdot T=\sum_{j=1}^{\infty} T_{j} x_{j}: x \in A, T \in B\right\}$ is bounded in $Y$.

In particular, Example 15 is applicable to the spaces $c_{0}(X), l^{p}(X)(1 \leq$ $p \leq \infty), c s(X), b s(X)$ when $X$ is a normed, barrelled ( $A$ space) space (see Appendix $\mathrm{C}$ of [16] for the definitions and topologies of these spaces). It should be noted that there are non-complete, normed, barrelled $(A)$ spaces $X$ so the spaces above may fail to be complete.

In the case of scalar sequence spaces as in Example 2, the spaces $P_{j} E$ are trivially barrelled so if $E$ has 0 -GHP, then $\sigma\left(E^{\beta}, E\right)$ bounded subsets are uniformly bounded on bounded subsets of $E$. Therefore, if $E^{\prime}=E^{\beta}$, then $E$ is a Banach-Mackey space ([18] 10.4) in this case, and if $E$ is also normed, $E$ is barrelled. These statements are similar to those in Theorem 1 of [12] and Corollaries 1 and 2 of [WCL] where different assumptions are made.

From Theorem 13 and Example 10, we have a uniform boundedness result for the Pettis integral.

Corollary 16. Suppose $\mu$ is $\sigma$-finite with $S=\cup_{j=1}^{\infty} A_{j}, \mu\left(A_{j}\right)<\infty$. Let $A \subset L^{\infty}(\mu)$ be bounded and $B \subset P(\mu, X)$ be $w\left(P(\mu, X), L^{\infty}(\mu)\right)$ bounded . Then $H=\left\{\int_{S} g f d \mu: g \in A, f \in B\right\}$ is bounded.

Proof. If $f \in P(\mu, X), g \in L^{\infty}(\mu)$, then

$$
f \cdot g=\sum_{j=1}^{\infty}\left\langle P_{j} f, g\right\rangle=\sum_{j=1}^{\infty} \int_{A_{j}} g f d \mu=\int_{S} g f d \mu .
$$


It follows from Corollary 16 that if $B \subset P(\mu, X)$ is pointwise bounded on $L^{\infty}(\mu)$, then

$$
\sup _{f \in B} \sup \left\{\left\|\int_{C} f d \mu\right\|: C \in \Sigma\right\}<\infty .
$$

The expression $\sup \left\{\left\|\int_{C} f d \mu\right\|: C \in \Sigma\right\}=\|f\|^{\prime}$ defines a norm on $P(\mu, X)$ which is equivalent to the norm previously defined ([11] p.198) so subsets of $P(\mu, X)$ which are pointwise bounded on $L^{\infty}(\mu)$ are norm bounded, a conclusion like that of the classical Uniform Boundedness Principle. Recall the space of Pettis integrable functions is not complete but is barrelled $([5])$.

We next establish several uniform convergence results for abstract duality pairs with projections.

Theorem 17. Assume that $E$ has 0-GHP. If $y \in E^{\beta}$ and $x^{i} \rightarrow 0$ in $E$, then the series $\sum_{j=1}^{\infty}\left\langle P_{j} x^{i}, y\right\rangle$ converge uniformly for $i \in \mathbf{N}$.

Proof. If the conclusion fails, there exists a symmetric neighborhood of $0, U$, in $G$ such that for every $k$ there exist $m_{k}>k, p_{k}$ such that $\sum_{j=m_{k}}^{\infty}\left\langle P_{j} x^{p_{k}}, y\right\rangle \notin U$. Choose a symmetric neighborhood $V$ such that $V+$ $V \subset U$. For $k=1$ let $m_{1}$ and $p_{1}$ satisfy this condition so $\sum_{j=m_{1}}^{\infty}\left\langle P_{j} x^{p_{1}}, y\right\rangle \notin$ $U$. There exists $n_{1}>m_{1}$ such that $\sum_{j=n_{1}+1}^{\infty}\left\langle P_{j} x^{p_{1}}, y\right\rangle \in V$. Then $\sum_{j=m_{1}}^{n_{1}}\left\langle P_{j} x^{p_{1}}, y\right\rangle \notin V$. There exists $N_{1}$ such that $\sum_{j=m}^{n}\left\langle P_{j} x^{i}, y\right\rangle \in V$ for $1 \leq i \leq p_{1}, n>m \geq N_{1}$. Let $p_{2}, m_{2}>N_{1}, n_{2}>m_{2}$ satisfy the condition above for $k=N_{1}$ so

$$
\sum_{j=m_{2}}^{n_{2}}\left\langle P_{j} x^{p_{2}}, y\right\rangle \notin V
$$

(this is an abuse of the notation above but avoids multiple subscripts, should cause no difficulty and makes the notation more palatable). Then $p_{2}>p_{1}$ by the choice of $N_{1}$. Continuing this construction produces increasing sequences $\left\{p_{k}\right\},\left\{m_{k}\right\},\left\{n_{k}\right\}, m_{k+1}>n_{k}>m_{k}$ with

$$
(*) \sum_{j=m_{k}}^{n_{k}}\left\langle P_{j} x^{p_{k}}, y\right\rangle \notin V .
$$

Set $I_{k}=\left[m_{k}, n_{k}\right]$ so $\left\{I_{k}\right\}$ is an increasing sequence of intervals. Since $x^{k} \rightarrow 0$, the 0 -GHP implies there exists a subsequence $\left\{q_{k}\right\}$ of $\left\{p_{k}\right\}$ such that

$$
x=\sum_{k=1}^{\infty} P_{I_{q_{k}}} x^{q_{k}} \in E,
$$


where the series is $w\left(E, E^{\beta}\right)$ convergent. Condition $\left(^{*}\right)$ implies the series

$$
\sum_{j=1}^{\infty}\left\langle P_{j} x, y\right\rangle
$$

doesn't converge which gives the desired contradiction.

A similar result for sectional operators was established in Theorem 12 of [20]; see also Theorem 2.22 of [16] for a scalar sequence version. Even in the case of scalar sequence spaces the conclusion of Theorem 17 may fail to hold without some assumptions on the space (Example 2.23 of [16]).

We now continue to establish other uniform convergence results which require different gliding hump assumptions.

Definition 18. The space $E$ (or the triple $(E, F: G)$ ) has the signed weak gliding hump property (signed WGHP) if whenever $x \in E$ and $\left\{I_{k}\right\}$ is an increasing sequence of intervals, there exist a subsequence $\left\{p_{k}\right\}$ and a sequence of signs $\left\{s_{k}\right\}$ such that the series $\sum_{k=1}^{\infty} s_{k} P_{I_{p_{k}}} x$ is $w\left(E, E^{\beta}\right)$ convergent in $E$. If all of the signs can be chosen equal to $1, E$ is said to have the weak gliding hump property (WGHP).

Remark 19. See [16], Appendices $B$ and $C$, for the sequence space definitions and examples where as noted earlier the series $\sum_{k=1}^{\infty} s_{k} P_{I_{p_{k}}} x$ is required to converge pointwise, an option not available in this abstract setting. In [20] there is a similar definition. Note that the signed-WGHP does not depend on the topology of $E$ but on the topology $w\left(E, E^{\beta}\right)$; the signed-WGHP is an algebraic condition.

We give a condition which is sufficient for a triple to have WGHP.

Notation 20. If $I \subset \mathbf{N}$ is an infinite set whose elements are arranged in a sequence $\left\{n_{j}\right\}$ and $\left\{x_{j}\right\} \subset E$, we write $\sum_{j \in I} x_{j}=\sum_{j=1}^{\infty} x_{n_{j}}$ provided the series $\sum_{j=1}^{\infty} x_{n_{j}}$ is $w\left(E, E^{\beta}\right)$ convergent to an element of $E$.

Definition 21. The space $E$ (or the triple $(E, F: G)$ ) is monotone if for every $x \in E$ and $I \subset \mathbf{N}$ the series $\sum_{j \in I} P_{j} x$ is $w\left(E, E^{\beta}\right)$ convergent to an element in $E$, denoted by $P_{I} x$.

Remark 22. A scalar (or vector) sequence space $\lambda$ is monotone if $\chi_{I} x \in \lambda$ when $x \in \lambda$ and $I \subset \mathbf{N}$, where $\chi_{I} x$ is the coordinate product of $\chi_{I}$ and $x$. This means the series $\sum_{j \in I} x_{j} e^{j}$ is coordinatewise convergent to $\chi_{I} x$. If the element $\chi_{I} x \in \lambda$, then the series $\sum_{j \in I} x_{j} e^{j}$ is $\sigma\left(\lambda, \lambda^{\beta}\right)$ convergent to $\chi_{I} x$ so the definition above agrees with the scalar (vector) definition of monotone. 
Examples of monotone sequence spaces are given in [16].

As is in the sequence space case, a monotone space has WGHP.

Proposition 23. If $E$ is monotone, then $E$ has WGHP.

Proof. Suppose $x \in E$ and $\left\{I_{j}\right\}$ is an increasing sequence of intervals. If $I=\cup_{j=1}^{\infty} I_{j}$, then

$$
P_{I} x=\sum_{k=1}^{\infty} \sum_{j \in I_{k}} P_{j} x=\sum_{k=1}^{\infty} P_{I_{k}} x
$$

is $w\left(E, E^{\beta}\right)$ convergent to an element of $E$.

Example 24. The triple $\left(L^{1}(\mu, X), L^{\infty}(\mu): X\right)$ of Example 5 is monotone and, therefore, has WGHP. Suppose $f \in L^{1}(\mu, X)$ and $I \subset \mathbf{N}$. Let $h$ be the pointwise limit of the series $\sum_{j \in I} P_{j} f=\sum_{j \in I} \chi_{A_{j}} f$. We claim $P_{I} f=h$. For this, let $g \in L^{\infty}(\mu)=L^{1}(\mu, X)^{\beta}$. Then, by countable additivity,

$$
\sum_{j \in I} P_{j} f \cdot g=\sum_{j \in I} \int_{A_{j}} g f d \mu=\int_{\cup_{j \in I} A_{j}} g f d \mu=\int_{S} g h d \mu
$$

justifying the claim. Similarly, $\left(L^{\infty}(\mu), L^{1}(\mu, X): X\right)$ and $\left(L^{p}(\mu, X)\right.$, $\left.L^{q}\left(\mu, X^{\prime}\right)\right)$ are monotone and have WGHP.

Example 25. The abstract triple $\left(L^{\infty}(\mu), P(\mu, X): X\right)$ with the projections $P_{j} g=\chi_{A_{j}} g$ in Example 6 is monotone. Note $L^{\infty}(\mu)^{\beta}=P(\mu, X)$ by the countable additivity of the Pettis integral ([4]II.3.5). We show that $L^{\infty}(\mu)$ is monotone and so has WGHP . Let $I=\left\{n_{j}\right\} \subset \mathbf{N}$ and $g \in L^{\infty}(\mu)$. Let $h$ be $\chi_{\cup_{j=1}^{\infty} A_{n_{j}}} g$. We claim that the series $\sum_{j=1}^{\infty} P_{n_{j}} g=\sum_{j=1}^{\infty} \chi_{A_{n_{j}}} g$ is $w\left(L^{\infty}(\mu), P(\mu, X)\right)$ convergent to $h$. By the countable additivity of the Pettis integral, if $f \in P(\mu, X)$, then

$$
\sum_{j=1}^{\infty} P_{n_{j}} g \cdot f=\sum_{j=1}^{\infty} \int_{A_{n_{j}}} g f d \mu=\int_{\cup_{j=1}^{\infty} A_{n_{j}}} g f d \mu=\int_{S} h f d \mu
$$

justifying the claim. Thus, $L^{\infty}(\mu)$ is monotone and has WGHP. The same proof shows that the triple $\left(P(\mu, X), L^{\infty}(\mu): X\right)$ is monotone.

Further examples of monotone spaces other than sequence spaces will be given later.

We establish a uniform convergence result for triples with signed WGHP.

Theorem 26. Assume $E$ has signed-WGHP. If $\left\{y^{k}\right\} \subset E^{\beta}$ is such that $\lim _{k}\left\langle x, y^{k}\right\rangle$ exists for each $x \in E$, then for each $x$ the series $\sum_{j=1}^{\infty}\left\langle P_{j} x, y^{k}\right\rangle$ converge uniformly for $k \in \mathbf{N}$. 
Proof. If the conclusion fails, there is a symmetric neighborhood of 0 , $U$, in $G$ such that for every $k$ there exist $p_{k}, n_{k}>m_{k}>k$ such that

$$
\text { (*) } \sum_{j=m_{k}}^{n_{k}}\left\langle P_{j} x, y^{p_{k}}\right\rangle \notin U .
$$

For $k=1$ this condition implies there exist $p_{1}, n_{1}>m_{1}>1$ such that $\sum_{j=m_{1}}^{n_{1}}\left\langle P_{j} x, y^{p_{1}}\right\rangle \notin U$. There exists $m^{\prime}>n_{1}$ such that $\sum_{j=m}^{n}\left\langle P_{j} x, y^{k}\right\rangle \in U$ for $1 \leq k \leq p_{1}, n>m>m^{\prime}$. The condition $(*)$ for $k=m^{\prime}$ implies there exist $p_{2}, n_{2}>m_{2}>m^{\prime}$ such that $\sum_{j=m_{2}}^{n_{2}}\left\langle P_{j} x, y^{p_{2}}\right\rangle \notin U$. Then $p_{2}>p_{1}$. Continuing this construction produces increasing sequences $\left\{p_{k}\right\},\left\{m_{k}\right\},\left\{n_{k}\right\}$ with $m_{k+1}>n_{k}>m_{k}$ and

$$
(* *) \quad \sum_{j=m_{k}}^{n_{k}}\left\langle P_{j} x, y^{p_{k}}\right\rangle \notin U .
$$

Set $I_{k}=\left[m_{k}, n_{k}\right]$ so $\left\{I_{k}\right\}$ is an increasing sequence of intervals. Define a matrix

$$
M=\left[m_{i j}\right]=\left[\sum_{l \in I_{j}}\left\langle P_{l} x, y^{p_{i}}\right\rangle\right] .
$$

We claim that $M$ is a signed $K$-matrix. First, the columns of $M$ converge by hypothesis. Next, given any subsequence there exist a further subsequence $\left\{r_{j}\right\}$ and signs $\left\{s_{j}\right\}$ such that the series $\sum_{j=1}^{\infty} s_{j} P_{I_{r_{j}}} x=z$ is $w\left(E, E^{\beta}\right)$ convergent in $E$. Then

$$
\sum_{j=1}^{\infty} s_{j} m_{i r_{j}}=\sum_{j=1}^{\infty} s_{j} \sum_{l \in I_{r_{j}}}\left\langle P_{l} x, y^{p_{i}}\right\rangle=\left\langle z, y^{p_{i}}\right\rangle
$$

and $\left\{\left\langle z, y^{p_{i}}\right\rangle\right\}$ converges in $G$ by hypothesis. Hence, $M$ is a signed $K$ matrix and the diagonal of $M$ converges to 0 by the signed version of the Antosik-Mikusinski Matrix Theorem. But, this contradicts (**).

A similar result was established in Theorem 5 of [20]; see also Theorems 2.26 and 11.14 of [16] for the sequence space result. The results in [16] were used to establish the weak sequential completeness of $\beta$-duals (see also [14]). Again without assumptions on the space $E$ the conclusion of Theorem 26 may fail ([16], Example 2.30).

The results in Theorems 17 and 26 require different gliding hump assumptions and these gliding hump assumptions are independent of one another; the space $c$ has 0 -GHP but not WGHP while the space $c_{00}$ has WGHP but not 0-GHP (see Proposition B.29 of [16] for a relationship). 
Using Theorems 17 and 26 we can obtain a more general result for spaces with both 0-GHP and signed-WGHP.

Theorem 27. Assume $E$ has 0-GHP and signed-WGHP. If $\left\{y^{k}\right\} \subset E^{\beta}$ is such that $\lim _{k}\left\langle x, y^{k}\right\rangle$ exists for each $x \in E$ and $x^{k} \rightarrow 0$ in $E$, then the series $\sum_{j=1}^{\infty}\left\langle P_{j} x^{k}, y^{l}\right\rangle$ converge uniformly for $k, l \in \mathbf{N}$.

Proof. If the conclusion fails, as in the proof above, there exists a neighborhood, $U$, of 0 in $G$ such that for every $k$ there exist $k<m_{k}<n_{k}, p_{k}, q_{k}$ such that $\sum_{j=m_{k}}^{n_{k}}\left\langle P_{j} x^{p_{k}}, y^{q_{k}}\right\rangle \notin U$. By this condition for $k=1$ there exist $p_{1}, q_{1}, n_{1}>m_{1}>1$ such that $\sum_{j=m_{1}}^{n_{1}}\left\langle P_{j} x^{p_{1}}, y^{q_{1}}\right\rangle \notin U$. Now by Theorems 17 and 26 above there exists $m^{\prime}>n_{1}$ such that $\sum_{j=p}^{q}\left\langle P_{j} x^{k}, y^{l}\right\rangle \in U$ for $k \in$ $\mathbf{N}, 1 \leq l \leq q_{1}$ and $1 \leq k \leq p_{1}, l \in \mathbf{N}, q>p>m^{\prime}$. By the condition above for $k=m^{\prime}$ there exist $p_{2}, q_{2}, n_{2}>m_{2}>m^{\prime}$ such that $\sum_{j=m_{2}}^{n_{2}}\left\langle P_{j} x^{p_{2}}, y^{q_{2}}\right\rangle \notin$ $U$. By the choice of $m^{\prime}$ we have $p_{2}>p_{1}, q_{2}>q_{1}$. Continuing this construction produces increasing sequences $\left\{p_{k}\right\},\left\{q_{k}\right\},\left\{m_{k}\right\},\left\{n_{k}\right\}, m_{k+1}>n_{k}>$ $m_{k}$ with

$$
\text { (\#) } \sum_{j=m_{k}}^{n_{k}}\left\langle P_{j} x^{p_{k}}, y^{q_{k}}\right\rangle \notin U .
$$

Set $I_{k}=\left[m_{k}, n_{k}\right]$ so $\left\{I_{k}\right\}$ is an increasing sequence of intervals. Define a matrix

$$
M=\left[m_{i j}\right]=\left[\sum_{l=m_{j}}^{n_{j}}\left\langle P_{l} x^{p_{j}}, y^{q_{i}}\right\rangle\right]=\left[\left\langle P_{I_{j}} x^{p_{j}}, y^{q_{i}}\right\rangle\right] .
$$

We claim that $M$ is a $K$-matrix. First the columns of $M$ converge by hypothesis. Next, by 0-GHP, given any increasing sequence of integers, there is a subsequence $\left\{r_{k}\right\}$ such that the series $x=\sum_{k=1}^{\infty} P_{I_{r_{k}}} x^{p_{r_{k}}}$ is $w\left(E, E^{\beta}\right)$ convergent in $E$. Then

$$
\sum_{k=1}^{\infty} m_{i r_{k}}=\sum_{k=1}^{\infty}\left\langle P_{I_{r_{k}}} x^{p_{r_{k}}}, y^{q_{i}}\right\rangle=\left\langle x, y^{q_{i}}\right\rangle
$$

and $\left\{\left\langle x, y^{q_{i}}\right\rangle\right\}$ converges. Hence, $M$ is a $K$-matrix and by the AntosikMikusinski Matrix Theorem the diagonal of $M$ converges to 0. But this contradicts (\#).

There is a version of this result for scalar sequences given in Theorem 2.39 of [16] where the hypothesis that $\lambda$ has signed WGHP is needed.

We give an application of Theorem 27 to weak convergence in $L^{\infty}(\mu)$. 
Corollary 28. Suppose $\left\{g_{k}\right\} \subset L^{\infty}(\mu)$ is such that $\lim \int_{S} g_{k} f d \mu$ exists for every $f \in L^{1}(\mu, X)$. Then if $\left\{f_{k}\right\} \subset L^{1}(\mu, X)$ converges to 0 in $L^{1}(\mu, X)$, the family of vector measures $H=\left\{\int g_{k} f_{j} d \mu: j, k \in \mathbf{N}\right\}$ is uniformly countably additive.

Proof. This follows from Theorem 27 and Examples 9 and 24 applied to the triple $\left(L^{1}(\mu, X), L^{\infty}(\mu): X\right)$ since we can take $\left\{A_{j}\right\}$ to be an arbitrary pairwise disjoint sequence from $\Sigma$.

From Examples 9 and 24 and Theorem 27, we also have a dual result for sequences $\left\{f_{k}\right\} \subset L^{1}(\mu, X)$ such that $\lim \int_{S} g f_{k} d \mu$ exists for every $g \in$ $L^{\infty}(\mu)$ and $g_{k} \rightarrow 0$ in $L^{\infty}(\mu)$.

A similar result holds for the triple $\left(L^{p}(\mu, X), L^{q}\left(\mu, X^{\prime}\right)\right)$ (Examples 9 and 24, Theorem 27).

We give a similar application of Theorem 27 to weak convergence in the space of Pettis integrable functions (Example 6).

Corollary 29. Suppose $\left\{f_{k}\right\} \subset P(\mu, X)$, the space of Pettis integrable functions (Example 6), is such that $\lim _{k} \int_{S} g f_{k} d \mu$ exists for every $g \in$ $L^{\infty}(\mu)$. Then if $g_{k} \rightarrow 0$ in $L^{\infty}(\mu)$, the family of vector measures $H=$ $\left\{\int g_{j} f_{k} d \mu: j, k \in \mathbf{N}\right\}$ is uniformly countably additive.

Proof. This follows from Examples 10 and 25 and Theorem 27.

A similar dual result can be established for weak convergence in the triple $\left(P(\mu, X), L^{\infty}(\mu): X\right)$. By Examples 11 and 25 and Theorem 27 if the family $\left\{g_{k}\right\} \subset L^{\infty}(\mu)$ is such that $\lim \int_{S} g_{k} f d \mu$ exists for every $f \in P(\mu, X)$ and $f_{k} \rightarrow 0$ in $P(\mu, X)$, then the family of vector measures $H=\left\{\int g_{k} f_{j} d \mu\right.$ : $j, k \in \mathbf{N}\}$ is uniformly countably additive.

The conclusions in Corollaries 28 and 29 and the observation above also imply that the families $H$ in the conclusions are uniformly $\mu$ continuous. This follows from Theorem 3.14.1 of [17] since each indefinite Pettis integral is $\mu$ continuous. See [6] IV.8.9, IV.8.11 and IV.9.1 for applications of uniform countable additivity and uniform $\mu$-continuity.

We give an example of a space of measures with WGHP and 0-GHP and give an application of Theorem 27 . Let $(S, \Sigma, \mu)$ be a measure space with $\left\{A_{j}\right\}$ a pairwise disjoint sequence from $\Sigma$.

Example 30. Let $B(\Sigma)$ be the space of all bounded, $\Sigma$-measurable functions defined on $S$ with the sup-norm. Let $X$ be a Banach space and let $c a(\Sigma, X: \mu)$ be the space of all countably additive set functions $\nu: \Sigma \rightarrow X$ 
which are $\mu$ continuous (i.e., $\lim _{\mu(A) \rightarrow 0} \nu(A)=0$ ). We define a complete norm on $c a(\Sigma, X: \mu)$ by setting $\|\nu\|=\sup \{\|\nu(A)\|: A \in \Sigma\}$ (there is an equivalent norm using the semi-variation of $\nu$; see [6]IV.10.4). Then $(c a(\Sigma, X: \mu), B(\Sigma): X)$ is an abstract triple with respect to the pairing $\langle\nu, f\rangle=\int_{S} f d \nu$ (see [6] IV.10 for integration of scalar functions with respect to vector valued measures especially $I V .10 .8(c)$ for integrating bounded functions). Define projections $P_{j}$ on $(c a(\Sigma, X: \mu), B(\Sigma): X)$ by $P_{j} \nu(\cdot)=\nu\left(A_{j} \cap \cdot\right)$. We also have $c a(\Sigma, X: \mu)^{\beta}=B(\Sigma)$ by the countable additivity of the integral $([6] I V .10 .8(d))$. We claim that $(c a(\Sigma, X$ : $\mu), B(\Sigma): X)$ is monotone and, therefore, has WGHP. Let $\nu \in c a(\Sigma, X: \mu)$ and $I=\left\{n_{j}\right\} \subset \mathbf{N}$. If $f \in B(\Sigma)$, by the countable additivity of the integral,

$$
\sum_{j \in I}\left\langle P_{j} \nu, f\right\rangle=\sum_{j=1}^{\infty} \int_{A_{n_{j}}} f d \nu=\int_{\cup_{j=1}^{\infty} A_{n_{j}}} f d \nu
$$

Thus, the series

$$
\sum_{j \in I} P_{j} \nu
$$

converges in $w(c a(\Sigma, X: \mu), B(\Sigma))$ to $P_{I} \nu=\nu\left(\left(\cup_{j=1}^{\infty} A_{n_{j}}\right) \cap \cdot\right)$ and $c a(\Sigma, X$ : $\mu)$ is monotone. We next claim that $(c a(\Sigma, X: \mu), B(\Sigma): X)$ has $0-G H P$. Suppose $\left\|\nu_{k}\right\| \rightarrow 0$ and $\left\{I_{k}\right\}$ is an increasing sequence of intervals. Pick $n_{k}$ such that $\left\|\nu_{n_{k}}\right\| \leq 1 / 2^{k}$. For every $A \in \Sigma$,

$$
\sum_{k=1}^{\infty}\left\|\nu_{n_{k}}(A)\right\| \leq \sum_{k=1}^{\infty}\left\|\nu_{n_{k}}\right\| \leq \sum_{k=1}^{\infty} 1 / 2^{k}<\infty
$$

so the series $\sum_{k=1}^{\infty} \nu_{n_{k}}(A)$ is absolutely convergent. Let $\nu(A)=\sum_{k=1}^{\infty} \nu_{n_{k}}(A)$. By the Vitali-Hahn-Saks Theorem ([4]I.4.10), $\nu$ is countably additive and $\mu$ continuous so $\nu \in c a(\Sigma, X: \mu)$. Moreover, if $f \in B(\Sigma)$,

$$
\left\|\sum_{k=N}^{\infty} \int_{S} f d \nu_{n_{k}}\right\| \leq \sum_{k=N}^{\infty}\left\|\int_{S} f d \nu_{n_{k}}\right\| \leq \sum_{k=N}^{\infty}\|f\|_{\infty} 4\left\|\nu_{n_{k}}\right\| \leq 4\|f\|_{\infty} \sum_{k=N}^{\infty} 1 / 2^{k}
$$

(see [6]IV.10.8(c) and IV.10.4 for the inequality above) so the series

$$
\sum_{k=1}^{\infty} \int_{S} d \nu_{n_{k}}
$$

converges to $\nu$ in $w(c a(\Sigma, X: \mu), B(\Sigma))$. This establishes the claim. 
From Theorem 27 we have a result similar in spirit to the Nikodym Convergence Theorem ([6]IV10.6) except that we have a stronger hypothesis and a stronger conclusion.

Corollary 31. Suppose $\nu_{k} \rightarrow 0$ in $c a(\Sigma, X: \mu)$ and $\left\{f_{k}\right\} \subset B(\Sigma)$ is such that $\lim _{k} \int_{S} f_{k} d \nu$ exists for every $\nu \in c a(\Sigma, X: \mu)$. Then the family of vector measures $H=\left\{\int f_{k} d \nu_{l}: k, l \in \mathbf{N}\right\}$ is uniformly countably additive and uniformly $\mu$ continuous.

Proof. The first conclusion follows from Theorem 27. The last conclusion follows from Theorem 3.14.1 of [17].

Similarly, if $c a(\Sigma, X)$ is the space of countably additive, $X$ valued measures with the norm as defined above, $(c a(\Sigma, X), B(\Sigma): X)$ forms an abstract triple under the map $\langle\nu, f\rangle=\int_{S} f d \nu$ and with projections as defined above is monotone and has 0 -GHP (in the proof of 0-GHP one employs the Nikodym Convergence Theorem ([6]IV.10.6) in place of the Vitali-HahnSaks Theorem). Thus, a result analogous to Corollary 31 holds in this case.

The results in Theorems 17 and 26 give conditions for the series to converge uniformly over subsets of either $E$ or $E^{\beta}$. We can obtain a similar result where the series converge uniformly over subsets of both $E$ and $E^{\beta}$ as in Theorem 27 by imposing another gliding hump condition.

Definition 32. The space $E$ (or the triple $(E, F: G)$ ) has the signed strong gliding hump property (signed SGHP) if whenever $\left\{x^{k}\right\}$ is bounded in $E$ and $\left\{I_{k}\right\}$ is an increasing sequence of intervals, there exist a subsequence $\left\{p_{k}\right\}$ and a sequence of signs $\left\{s_{k}\right\}$ such that the series $\sum_{k=1}^{\infty} s_{k} P_{I_{p_{k}}} x^{p_{k}}$ is $w\left(E, E^{\beta}\right)$ convergent in $E$. If all the signs can be chosen equal to1, then $E$ has the strong gliding hump property (SGHP).

Note that the SGHP depends on the duality triple but also on the topology of the space $E$. See [16] for the scalar and vector space definitions and examples. A different definition is given in [20] where the sequence $\left\{x^{k}\right\}$ is required to be $w\left(E, E^{\beta}\right)$ bounded.

Theorem 33. Assume $E$ has signed SGHP. If $y \in E^{\beta}$ and $B \subset E$ is bounded, then the series $\sum_{j=1}^{\infty}\left\langle P_{j} x, y\right\rangle$ converge uniformly for $x \in B$. 
Proof. If the conclusion fails, then as in previous arguments there exist a symmetric neighborhood $U$ in $G, m_{k+1}>n_{k}>m_{k}, x^{k} \in B$ such that

$$
\text { (*) } \quad \sum_{j=m_{k}}^{n_{k}}\left\langle P_{j} x^{k}, y\right\rangle \notin U .
$$

Put $I_{k}=\left[m_{k}, n_{k}\right]$. By the signed SGHP, there exist an increasing sequence $\left\{p_{k}\right\}$ and signs $\left\{s_{k}\right\}$ such that $x=\sum_{k=1}^{\infty} s_{k} P_{I_{p_{k}}} x^{p_{k}} \in E$ with the series being $w\left(E, E^{\beta}\right)$ convergent. But then the series

$$
\sum_{j=1}^{\infty}\left\langle P_{j} x, y\right\rangle=\sum_{k=1}^{\infty} s_{k} \sum_{j=m_{k}}^{n_{k}}\left\langle P_{j} x^{k}, y\right\rangle
$$

fails the Cauchy criterion by $(*)$.

A similar result is obtained in Theorem 8 of [20] under different hypothesis. See also Theorem 2.16 of [16].

Using Theorem 33 we can obtain a more general result where the series converge uniformly over subsets of both $E$ and $E^{\beta}$.

Theorem 34. Assume that $E$ has signed SGHP. If $\left\{y^{k}\right\} \subset E^{\beta}$ is such that $\lim _{k}\left\langle y^{k}, x\right\rangle$ exists for every $x \in E$ and $B \subset E$ is bounded, then the series $\sum_{j=1}^{\infty}\left\langle P_{j} x, y^{k}\right\rangle$ converge uniformly for $k \in \mathbf{N}, x \in B$.

Proof. If the conclusion fails, then as in previous arguments there exists a symmetric neighborhood $U$ in $G$ such that for every $k$ there exist $p_{k}>$ $k, n_{k}>m_{k}, x^{k} \in B$ such that

$$
\text { (\#) } \sum_{j=m_{k}}^{n_{k}}\left\langle P_{j} x^{k}, y^{p_{k}}\right\rangle \notin U .
$$

For $k=1$ this condition gives $\sum_{j=m_{1}}^{n_{1}}\left\langle P_{j} x^{1}, y^{p_{1}}\right\rangle \notin U$. By Theorem 33 there exists $m^{\prime}>n_{1}$ such that $\sum_{j=p}^{q}\left\langle P_{j} x, y^{k}\right\rangle \in U$ for $1 \leq k \leq p_{1}, x \in$ $B, q>p>m^{\prime}$. By (\#) for $k=m^{\prime}$ we have $\sum_{j=m_{2}}^{n_{2}}\left\langle P_{j} x^{2}, y^{p_{2}}\right\rangle \notin U$. Thus $p_{2}>p_{1}$. Continuing this construction produces increasing sequences $\left\{p_{k}\right\},\left\{m_{k}\right\},\left\{n_{k}\right\}, m_{k+1}>n_{k}>m_{k},\left\{x^{k}\right\} \subset B$ such that

$$
(\# \#) \sum_{j \in I_{k}}\left\langle P_{j} x^{k}, y^{p_{k}}\right\rangle \notin U,
$$

where $I_{k}=\left[m_{k}, n_{k}\right]$. Now define a matrix 


$$
M=\left[m_{i j}\right]=\left[\sum_{l \in I_{j}}\left\langle P_{l} x^{j}, y^{p_{i}}\right\rangle\right] .
$$

As in the proof of Theorem 26, $M$ is a signed $K$ matrix so by the signed version of the Antosik-Mikusinski Matrix Theorem the diagonal of $M$ converges to 0 contradicting (\#\#).

This result can be compared to Theorem 2.35 of [16]. The conclusion may fail to hold without assumptions on $E$ ([16], Example 2.20).

A uniform boundedness result as in Theorem 13 can be obtained from Theorem 34. Assume $E$ has signed SGHP and $P_{j} E$ is barrelled for every $j$. If $A \subset E$ is bounded and $B \subset E^{\beta}$ is pointwise bounded on $E$, then

$$
\left\{x \cdot y=\sum_{j=1}^{\infty}\left\langle P_{j} x, y\right\rangle: y \in B, x \in A\right\}
$$

is bounded. For suppose $x_{j} \in A, y_{j} \in B$ and $t_{j} \rightarrow 0$. Let $U$ be a neighborhood of 0 and $V$ a neighborhood of 0 such that $V+V \subset U$. Since $t_{k} y_{k} \rightarrow 0$ pointwise, by Theorem 34 there exists $N$ such that $\sum_{j=N}^{\infty} t_{k}\left\langle P_{j} x_{k}, y_{k}\right\rangle \in V$ for all $k$. Now $\left\{P_{j} x_{k}: k\right\}$ is bounded and $\left\{y_{k}\right\}$ is pointwise bounded so $\left\{\left\langle P_{j} x_{k}, y_{k}\right\rangle: k\right\}$ is bounded by the barrelledness assumption. Therefore, there exists $K$ such that $\sum_{j=1}^{N-1} t_{k}\left\langle P_{j} x_{k}, y_{k}\right\rangle \in V$ for $k \geq K$. Then if $k \geq K$,

$$
\sum_{j=1}^{\infty} t_{k}\left\langle P_{j} x_{k}, y_{k}\right\rangle=\sum_{j=1}^{N-1} t_{k}\left\langle P_{j} x_{k}, y_{k}\right\rangle+\sum_{j=N}^{\infty} t_{k}\left\langle P_{j} x_{k}, y_{k}\right\rangle \in V+V \subset U
$$

and the result follows. Note that the 0-GHP and SGHP assumptions are independent of one another (consider $c_{0}$ and $l^{\infty}$ ) so the result above and the result in Theorem 13 are independent.

We give an application of Theorem 34 to weak topologies on $L^{1}$. First we show $L^{\infty}(\mu)$ has SGHP in the triple $\left(L^{\infty}(\mu), L^{1}(\mu, X): X\right)$ when $L^{\infty}(\mu)$ has the essential-sup norm $\|\cdot\|_{\infty}$.

Example 35. $L^{\infty}(\mu)$ has SGHP in the triple $\left(L^{\infty}(\mu), L^{1}(\mu, X): X\right)$ when $L^{\infty}(\mu)$ has the essential-sup norm $\|\cdot\|_{\infty}$. First, note that $L^{\infty}(\mu)^{\beta}=L^{1}(\mu, X)$ by the countable additivity of the integral. Let $\left\{g_{k}\right\}$ be bounded in $L^{\infty}(\mu)$ and $\left\{I_{k}\right\}$ be an increasing sequence of intervals. For convenience, assume $\left\|g_{k}\right\|_{\infty} \leq 1$ for every $k$. The series $\sum_{k} P_{I_{k}} g_{k}=\sum_{k} \chi_{\cup_{j \in I_{k}} A_{j}} g_{k}$ converges pointwise to a function $g$ which is essentially bounded and measurable and 
so belongs to $L^{\infty}(\mu)$. We claim the series converges to $g$ with respect to $w\left(L^{\infty}(\mu), L^{1}(\mu, X)\right)$; this will establish the result. Let $f \in L^{1}(\mu, X)$. Then for every $n$, we have

$$
\left\|\sum_{k=1}^{n} \chi_{\cup_{j \in I_{k}} A_{j}} g_{k}(\cdot) f(\cdot)\right\| \leq\|f(\cdot)\|
$$

so by the Dominated Convergence Theorem

$$
\sum_{k=1}^{\infty} \int_{\cup_{j \in I_{k}} A_{j}} g_{k} f d \mu=\int_{S} g f d \mu
$$

so $g_{k} \rightarrow g$ in $w\left(L^{\infty}(\mu), L^{1}(\mu, X)\right)$.

We use Theorem 34 and Example 35 to establish a result for weak convergence in $L^{1}(\mu, X)$.

Theorem 36. Let $\left\{f_{k}\right\} \subset L^{1}(\mu, X)$ be such that $\lim \int_{S} g f_{k} d \mu$ exists for every $g \in L^{\infty}(\mu)$, i.e., $\left\{f_{k}\right\}$ is "weak" Cauchy. If $B \subset L^{\infty}(\mu)$ is bounded, then the family of vector measures $H=\left\{\int . g f_{k} d \mu: k \in \mathbf{N}, g \in B\right\}$ is uniformly countably additive.

This result should be compared to Theorem IV.8.9 of [6] which implies that if $\left\{f_{k}\right\} \subset L^{1}(\mu)$ is a weak Cauchy sequence, then $\left\{\int f_{k} d \mu: k \in \mathbf{N}\right\}$ is uniformly countably additivity. In Theorem 36 the uniform countable additivity is additionally over bounded subsets of $L^{\infty}(\mu)$. The conclusion of Theorem 36 can also be rephrased to read that the elements of the set $H$ are uniformly $\mu$ continuous (see [6], IV.8,[17]3.14.1).

We can also obtain results like those in Example 35 and Theorem 36 for vector and operator valued functions. Let $X, Y$ be Banach spaces and consider the pair $L^{\infty}(\mu, X), L^{1}(\mu, L(X, Y))$. If $f \in L^{1}(\mu, L(X, Y))$ and $g \in L^{\infty}(\mu, X)$, we first observe that the function $t \rightarrow f(t)(g(t))$ is strongly measurable. Suppose first that $g$ is a simple function, $g=\sum_{j=1}^{n} \chi_{B_{j}} x_{j}$, with $\left\{B_{j}\right\}, B_{j} \in \Sigma$, a partition of $S$. Then $f(\cdot)(g(\cdot))=\sum_{j=1}^{n} \chi_{B_{j}}(\cdot) f(\cdot)\left(x_{j}\right)$ so $f(\cdot)(g(\cdot))$ is a measurable function. If $g \in L^{\infty}(\mu, X)$, there exists a sequence $\left\{g_{k}\right\}$ of simple functions which converges pointwise almost everywhere to $g\left([4]\right.$ II.1). Then $f(\cdot)\left(g_{k}(\cdot)\right) \rightarrow f(\cdot)(g(\cdot))$ almost everywhere so $f(\cdot)(g(\cdot))$ is measurable. Moreover,

$$
\|f(t)(g(t))\| \leq\|f(t)\|\|g(t)\| \leq\|g\|_{\infty}\|f(t)\|
$$


implies $f(\cdot)(g(\cdot))$ is Bochner integrable with

$$
\left\|\int_{S} f(\cdot)(g(\cdot)) d \mu\right\| \leq\|g\|_{\infty}\|f\|_{1} .
$$

Thus, $\left(L^{\infty}(\mu, X), L^{1}(\mu, L(X, Y)): Y\right)$ is an abstract triple under the mapping $(g, f) \rightarrow \int_{S} f(\cdot)(g(\cdot)) d \mu$ and if $\left\{A_{j}\right\} \subset \Sigma$ is pairwise disjoint, $P_{j} g=\chi_{A_{j}} g$ defines projections on $L^{\infty}(\mu, X)$. The proof of Example 35 shows that the triple

$$
\left(L^{\infty}(\mu, X), L^{1}(\mu, L(X, Y)): Y\right)
$$

has SGHP so a result as in Theorem 36 holds in this case.

It should also be noted that dually, we have the triple

$$
\left(L^{1}(\mu, X), L^{\infty}(\mu, L(X, Y)): Y\right)
$$

under the same type of mapping and projections and as in Example 9 and Example 24 the triple has 0-GHP and is monotone so a result like that stated following Corollary 28 holds for this triple. Similarly, if $1<p<\infty$ and $\frac{1}{p}+\frac{1}{q}=1$, then the triple $\left(L^{p}(\mu, X), L^{q}(L(X, Y)): Y\right)$ has 0 -GHP and is monotone so a result as stated in Corollary 28 also holds for this triple. Note that in general this triple does not have SGHP.

Theorem 34 can also be used to establish a Schur type result for $l^{1}(X)$ when $X$ is a Banach space. Recall the Schur Theorem asserts that a sequence in $l^{1}$ which is weakly convergent converges in norm and a normed space with this property is called a Schur space. Consider the dual pair $l^{\infty}\left(X^{\prime}\right), l^{1}(X)$ under the pairing $\langle x, y\rangle=\sum_{j=1}^{\infty}\left(x_{j}, y_{j}\right)$, where $x=\left\{x_{j}\right\} \in$ $l^{\infty}\left(X^{\prime}\right), y=\left\{y_{j}\right\} \in l^{1}(X)$ and $(\cdot, \cdot)$ is the duality between $X^{\prime}$ and $X$. The triple $\left(l^{\infty}\left(X^{\prime}\right), l^{1}(X)\right)$ with the projections as in Example 3 has SGHP so Theorem 34 applies. Suppose $y^{k} \rightarrow 0$ with respect to $\sigma\left(l^{1}(X), l^{\infty}\left(X^{\prime}\right)\right)$. We show that if $X$ is a Schur space, then $\left\|y^{k}\right\|_{1} \rightarrow 0$ so $l^{1}(X)$ is a "Schur type space". Let $\epsilon>0$ and $N$ be such that $\left|\sum_{j=N}^{\infty}\left\langle P_{j} x, y^{k}\right\rangle\right|=\left|\sum_{j=N}^{\infty}\left(x_{j}, y_{j}^{k}\right)\right|<$ $\epsilon$ for all $k$ and $\|x\|_{\infty} \leq 1$ (Theorem 34). For each $j, \lim _{k} y_{j}^{k}=0$ with respect

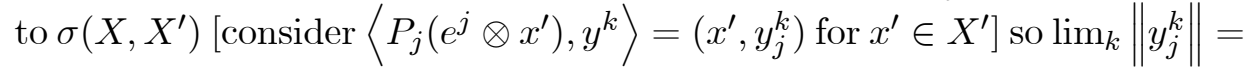
0 for each $j$. Therefore, there exists $M$ such that $\left|\sum_{j=1}^{N-1}\left(x_{j}, y_{j}^{k}\right)\right|<\epsilon$ for $\|x\|_{\infty} \leq 1$ and $k \geq M$. Hence, if $k \geq M$, then

$$
\left|\sum_{j=1}^{\infty}\left(x_{j}, y_{j}^{k}\right)\right|=\left|\sum_{j=1}^{N-1}\left(x_{j}, y_{j}^{k}\right)\right|+\left|\sum_{j=N}^{\infty}\left(x_{j}, y_{j}^{k}\right)\right|<2 \epsilon
$$


when $\|x\|_{\infty} \leq 1$. Fix $k \geq M$ and pick $x_{j}^{\prime} \in X^{\prime}$ such that $\left\|x_{j}^{\prime}\right\|=1$ and $\left|\left(x_{j}^{\prime}, y_{j}^{k}\right)\right|=\left\|y_{j}^{k}\right\|$ Then $x=\sum_{j=1}^{\infty} e^{j} \otimes x_{j}^{\prime} \in l^{\infty}\left(X^{\prime}\right),\|x\|_{\infty} \leq 1$, so

$$
\left|\sum_{j=1}^{\infty}\left\langle P_{j} x, y^{k}\right\rangle\right|=\left|\sum_{j=1}^{\infty}\left(x_{j}^{\prime}, y_{j}^{k}\right)\right|=\sum_{j=1}^{\infty}\left\|y_{j}^{k}\right\|=\left\|y^{k}\right\|_{1}<2 \epsilon
$$

for $k \geq M$.

A result for vector measures like that in Theorem 36 can also be obtained from Theorem 34 .

Example 37. Let $B(\Sigma)$ be the space of all bounded, $\Sigma$ measurable functions defined on $S$ with the sup-norm, $\|\cdot\|_{\infty}$ and let $c a(\Sigma, X)$ be the space of all countably additive set functions $\nu: \Sigma \rightarrow X$ with the complete norm $\|\nu\|=\sup \{\|\nu(A)\|: A \in \Sigma\}$ (there is an equivalent norm using the semivariation of $\nu$; see $[6] I V .10 .4)$. Then $(B(\Sigma), c a(\Sigma, X): X)$ forms an abstract triple under the pairing $\langle f, \nu\rangle=\int_{S} f d \nu$ (see [6]IV.10 for integration of scalar functions with respect to vector measures and particularly Theorem IV.10.8(c) where bounded measurable functions are integrable). Let $\left\{A_{j}\right\} \subset \Sigma$ be pairwise disjoint and define projections $P_{j}: B(\Sigma) \rightarrow B(\Sigma)$ by $P_{j} f=\chi_{A_{j}} f$ so $(B(\Sigma), c a(\Sigma, X): X)$ is a triple with projections when $B(\Sigma)$ has the sup-norm. Note $B(\Sigma)^{\beta}=c a(\Sigma, X)$ by the countable additivity of the elements of $c a(\Sigma, X)$. We show that $(B(\Sigma), c a(\Sigma, X): X)$ has SGHP. Suppose $\left\{f_{k}\right\}$ is a bounded subset of $B(\Sigma)$ and $\left\{I_{k}\right\}$ is an increasing sequence of intervals. For convenience set $B_{k}=\cup_{j \in I_{k}} A_{j}$ so $P_{I_{k}} f=\chi_{B_{k}} f$. The series $\sum_{k=1}^{\infty} P_{I_{k}} f_{k}=\sum_{k=1}^{\infty} \chi_{B_{k}} f_{k}$ converges pointwise to a function $f$ which is bounded and measurable and so belongs to $B(\Sigma)$. We claim the series $\sum_{k=1}^{\infty} P_{I_{k}} f_{k}$ is $w(B(\Sigma), c a(\Sigma, X))$ convergent to $f$. For this let $\nu \in c a(\Sigma, X)$. Then

$$
\sum_{k=1}^{\infty} P_{I_{k}} f_{k} \cdot \nu=\sum_{k=1}^{\infty} \int_{B_{k}} f_{k} d \nu=\int_{S} f d \nu
$$

by the Bounded Convergence Theorem (see Theorem IV.10.10 of [6]) justifying the claim.

From Theorem 34 and Example 37, we can obtain an improvement in the conclusion of the Nikodym Convergence Theorem ([6]IV.10.6).

Theorem 38. Let $\left\{\nu_{k}\right\} \subset c a(\Sigma, X)$ be such that $\lim _{k} \nu_{k}(A)=\nu(A)$ exists for every $A \in \Sigma$. If $B \subset B(\Sigma)$ is bounded, then the family of vector measures $H=\left\{\int . f d \nu_{k}: k \in \mathbf{N}, f \in B\right\}$ is uniformly countably additive. 
Proof. We claim that $\lim _{k} \int_{S} f d \nu_{k}$ exists for every $f \in B(\Sigma)$. Let $f \in B(\Sigma), \epsilon>0$ and pick a simple function $g$ such that $\|f-g\|_{\infty}<\epsilon$ and choose $n$ such that $k \geq n$ implies $\left\|\int_{S} g d \nu_{k}-\int_{S} g d \nu\right\|<\epsilon$. By the Nikodym Convergence Theorem $\nu$ is countably additive and by the Nikodym Boundedness Theorem, $\sup \left\{\left\|\nu_{k}\right\|: k \in \mathbf{N}\right\}=M<\infty$ ([6]IV.10.6, IV.9.8). Using the inequalities in IV.10.4 and IV.10.8(c) of [6], if $k \geq n$, we have

$$
\begin{aligned}
\left\|\int_{S} f d \nu_{k}-\int_{S} f d \nu\right\| & \leq\left\|\int_{S}(f-g) d \nu_{k}\right\|+\left\|\int_{S}(f-g) d \nu\right\|+\left\|\int_{S} g d \nu_{k}-\int_{S} g d \nu\right\| \\
& \leq 4\|f-g\|_{\infty}(M+\|\nu\|)+\left\|\int_{S} g d \nu_{k}-\int_{S} g d \nu\right\| \\
& <\epsilon(4(M+\|\nu\|)+1)
\end{aligned}
$$

justifying the claim. The result now follows from Theorem 34 and Example 37.

In the classical Nikodym Convergence Theorem ([6]IV.10.6) the uniform countable additivity is for the measures $\left\{\nu_{k}\right\}$ and in the result above the uniform additivity is for the indefinite integrals over bounded subsets of $B(\Sigma)$. For an application of uniform countable additivity in this setting, see [6]IV.13.22.

Theorem 38 also gives an improvement to the Vitali-Hahn-Saks Theorem ([6]IV.7.2). If each of the measures $\nu_{k}$ is $\mu$-continuous with respect to a positive measure $\mu$, then each indefinite integral in $H$ is $\mu$ continuous so the family of indefinite integrals in $H$ is uniformly $\mu$ continuous by Theorem 38 and Theorem 3.14.1 of [17] ( if $\nu \in c a(\Sigma, X)$ is $\mu$ continuous and $g$ is simple, then clearly $\int . g d \nu$ is $\mu$ continuous, and if $f \in B(\Sigma)$, then $\int . f d \nu$ is the pointwise limit of a sequence $\int g_{k} d \nu$ of indefinite integrals of simple functions $g_{k}$ so $\int f d \nu$ is $\mu$ continuous by the Vitali-Hahn-Saks Theorem ([6]IV.7.2)).

We can also obtain a result similar to Theorem 38 for operator valued measures and vector valued functions. Let $X, Y$ be Banach spaces, $B(\Sigma, X)$ the space of all bounded, $X$ valued, $\Sigma$ measurable functions with the supnorm, $\lambda: \Sigma \rightarrow[0, \infty)$ a finite measure, and $c a(\Sigma, L(X, Y): \lambda)$ the space of all $\nu: \Sigma \rightarrow L(X, Y)$ which are countably additive and $\lambda$ continuous. The space $c a(\Sigma, L(X, Y): \lambda)$ is given the (operator) semi-variation norm $\|\nu\|$ (see [1]1). Then $(B(\Sigma, X), c a(\Sigma, L(X, Y): \lambda): Y)$ forms an abstract triple under the continuous bilinear map $(f, \nu) \rightarrow \int_{S} f d \nu$ (for the integration of vector valued functions with respect to operator valued measures and their properties, see Bartle $([1]))$ and projections $P_{j}$ can be defined as above. Under these assumptions, the integral is countably additive, bounded mea- 
surable functions are integrable and the Bounded Convergence Theorem holds for the integral. Hence, the proof of Example 37 can be repeated to show that the triple $(B(\Sigma, X), c a(\Sigma, L(X, Y): \lambda): Y)$ has SGHP and Theorem 34 gives a result like Theorem 38 .

Theorem 39. Let $\left\{\nu_{k}\right\} \subset c a(\Sigma, L(X, Y): \lambda)$ be such that $\lim _{k} \int_{S} f d \nu_{k}$ exists for every $f \in B(\Sigma, X)$. If $B \subset B(\Sigma, X)$ is bounded, then the family of $Y$ valued measures $H=\left\{\int f d \nu_{k}: k \in \mathbf{N}, f \in B\right\}$ is uniformly countably additive.

Finally, we consider spaces of integrable functions with respect to a measure with values in a locally convex space. Assume $G$ is a sequentially complete Hausdorff locally convex space and $\nu: \Sigma \rightarrow G$ is a countably additive vector measure. A $\Sigma$ measurable function $f: S \rightarrow \mathbf{R}$ is scalarly integrable with respect to $\nu$ if $f$ is $x^{\prime} \nu$ integrable for every $x^{\prime} \in G^{\prime}$ and $f$ is $\nu$ integrable if $f$ is scalarly $\nu$ integrable and for every $A \in \Sigma$ there exists $x_{A} \in G$ such that $\int_{A} f d x^{\prime} \nu=x^{\prime}\left(x_{A}\right)$; we write $\int_{A} f d \nu=x_{A}$. (For the integral and for properties of the integral, we refer to [8]; see also, [13].) Let $L^{1}(\nu)$ be the space of all $\nu$ integrable functions. We will describe the topology of $L^{1}(\nu)$. Let $P$ be a family of semi-norms which generate the topogy of $G$ and if $p \in P$, let $U_{p}=\{x \in G: p(x) \leq 1\}$ and $U_{p}^{0}$ be the polar of $U_{p}$. Define a semi-norm $\widehat{p}$ on $L^{1}(\nu)$ by

$$
\widehat{p}(f)=\left\{\int_{S}|f| d\left|x^{\prime} \nu\right|: x^{\prime} \in U_{p}^{0}\right\}
$$

the topology of $L^{1}(\nu)$ is defined to be the topology generated by the seminorms $\{\hat{p}: p \in P\}$. Since $G$ is sequentially complete, the product of bounded measurable functions and $\nu$ integrable functions are $\nu$ integrable ([8] Theorem II.3.1) and we can define an abstract triple $\left(B(\Sigma), L^{1}(\nu): G\right)$ under the integration map $(f, g) \rightarrow \int_{S} f g d \nu$ when $B(\Sigma)$ has the sup-norm and this bilinear map is continuous since $p\left(\int_{S} f g d \nu\right) \leq\|f\|_{\infty} \widehat{p}(g)$. The Dominated Convergence Theorem holds for the integral ([8] Theorem II.4.2) so the proof in Example 35 shows that the triple $\left(B(\Sigma), L^{1}(\nu): G\right)$ with the projections defined as before has SGHP and a weak convergence result as in Theorem 36 holds for the space $L^{1}(\nu)$.

Corollary 40. Suppose $\left\{f_{k}\right\} \subset L^{1}(\nu)$ is such that $\lim _{k} \int_{S} f_{k} g d \nu$ exists for every $g \in B(\Sigma)$ and $B \subset B(\Sigma)$ is bounded. Then the family of vector measures $H=\left\{\int f_{k} g d \nu: k \in \mathbf{N}, g \in B\right\}$ is uniformly countably additive. 
Dually, consider the triple $\left(L^{1}(\nu), B(\Sigma): G\right)$ under the integration map. Since the indefinite integral is countably additive, the proof in Example 24 shows that the triple is monotone and, therefore, has WGHP. We also consider the 0-GHP for the triple. In order to do this we first establish an abstract result for triples and then apply the result to $\left(L^{1}(\nu), B(\Sigma): G\right)$.

Theorem 41. Let $(E, F: G)$ be an abstract triple with projections $\left\{P_{j}\right\}$ and $E$ a complete, metrizable locally convex space whose topology is generated by the semi-norms $p_{1} \leq p_{2} \leq \ldots$. Assume that $p_{l}\left(P_{I} x\right) \leq p_{l}(x)$ for every $l \in \mathbf{N}$ and finite $I \subset \mathbf{N}$. Then $E$ has $0-G H P$.

Proof. Let $x_{k} \rightarrow 0$ in $E$ and $\left\{I_{k}\right\}$ be an increasing sequence of intervals. Pick an increasing sequence of integers $\left\{n_{k}\right\}$ such that $p_{n_{k}}\left(x_{j}\right)<1 / 2^{k}$ for $j \geq n_{k}$. Consider the series $\sum_{k=1}^{\infty} P_{I_{n_{k}}} x_{n_{k}}$. We claim the series is absolutely convergent in $E$. Fix $l \in \mathbf{N}$. Then by hypothesis

$$
\sum_{\left\{k: n_{k} \geq l\right\}} p_{l}\left(P_{I_{n_{k}}} x_{n_{k}}\right) \leq \sum_{\left\{k: n_{k} \geq l\right\}} p_{n_{k}}\left(P_{I_{n_{k}}} x_{n_{k}}\right) \leq \sum_{\left\{k: n_{k} \geq l\right\}} p_{n_{k}}\left(x_{n_{k}}\right) \leq \sum_{\left\{k: n_{k} \geq l\right\}} 1 / 2^{k}
$$

so the series is absolutely convergent and, therefore, convergent to some $x$ in $E$ since $E$ is complete. Hence, the series $\sum_{k=1}^{\infty} P_{I_{n_{k}}} x_{n_{k}}$ is $w(E, F)$ convergent to $x$ and $E$ has 0 -GHP.

If $G$ is sequentially complete, metrizable, then $L^{1}(\nu)$ is complete (see [8] IV4.1 and IV.7.1 for this result) and the projections satisfy the condition that $\widehat{p}\left(P_{I} f\right) \leq \widehat{p}(f)$ for $f \in L^{1}(\nu)$ and $I$ finite. Thus, the theorem above applies and the triple $\left(L^{1}(\nu), B(\Sigma): G\right)$ has 0 -GHP and a weak convergence result as in Theorem 27 holds in this case.

Corollary 42. Suppose $\left\{g_{k}\right\} \subset B(\Sigma)$ is such that $\lim _{k} \int_{S} f g_{k} d \nu$ exists for every $f \in L^{1}(\nu)$. If $f_{k} \rightarrow 0$ in $L^{1}(\nu)$, then the family of vector measures $H=\left\{\int f_{l} g_{k} d \nu: k, l \in \mathbf{N}\right\}$ is uniformly countably additive.

\section{References}

[1] R. Bartle, A general bilinear vector integral, Studia Math., 15, pp. 337-352, (1956).

[2] C. Bosch and C. Swartz, Functional Calculi, World Sci. Publ., Singapore, (2013). 
[3] C. Cho, R. Li and C. Swartz, Subseries convergence in abstract duality pairs, Proy. J. Math., 33, pp. 447-470, (2014).

[4] J. Diestel and J. Uhl, Vector Measures, Amer. Math. Soc. Surveys \#15, Providence, (1977).

[5] L. Drewnowski, M. Florencio and P. Paul, The Space of Pettis Integrable Functions is Barrelled, Proc, Amer. Math. Soc., 114, pp. 687694, (1992).

[6] N. Dunford and J. Schwartz, Linear Operators I, Interscience, N. Y., (1958).

[7] P. K. Kamthan and M. Gupta, Sequence Spaces and Series, Marcel Dekker, N. Y., (1981).

[8] I. Kluvanek and G. Knowles, Vector Measures and Control Systems, North-Holland, Amsterdam, (1976).

[9] Lee Peng Yee, Sequence Spaces and the Gliding Hump Property, Southeast Asia Bull. Math., Special Issue, pp. 65-72, (1993).

[10] Li Ronglu and C. Swartz, Spaces for Which the Uniform Boundedness Principle Holds, Studia Sci. Math. Hung., 27, pp. 379-384, (1992).

[11] K.Musial, Topics in the Theorey of Pettis Integration, Rend. Instituto Mat. Univ. Trieste, Vol. XXIII, (1991).

[12] D. Noll and W. Stadler, Abstract sliding hump techniques and characterizations of barrelled spaces, Studia Math., 94, pp. 103-120, (1989).

[13] T. V. Panchapagesan, The Bartle-Dunford-Schwartz Integral, Birkhauser, Basel, (2008).

[14] C. Stuart, Weak Sequential Completeness of $\beta$-duals in Sequence Spaces, Rocky Mount. Math. J., 26, pp. 1559-1568, (1996).

[15] C. Swartz, Infinite Matrices and the Gliding Hump, World Sci. Publ., Singapore, (1996).

[16] C. Swartz, Multiplier Convergent Series. World. Sci. Publ., Singapore, (2009).

[17] C. Swartz, Measure, Integration and Function Spaces, World Sci. Publ., Singapore, (1994). 
[18] A. Wilansky, Modern Methods in Topological Vector Spaces, McGrawHill, N. Y., (1978).

[19] Junde $\mathrm{Wu}$, Jianwen Luo and Chengri Cui, The Abstract Gliding Hump Properties and Applications, Taiwan. J. Math., 10, pp. 639649, (2006).

[20] Zheng Fu, Cui Chengri and Li Ronglu, Abstract Gliding Hump Properties in the Vector-Valued Dual Pair, Acta Anal. Funct. Appl., 12, pp. 322-327, (2010).

\section{Charles Swartz}

Mathematics Department

New Mexico State University

Las Cruces, NM 88003,

U. S. A.

e-mail : cswartz@nmsu.edu 